\title{
Variability in lung deposition of inhaled drug, within and between asthmatic patients, with a pMDI and a dry powder inhaler, Turbuhaler ${ }^{\circledR}$
}

\author{
Lars Borgström ${ }^{\mathrm{a}, *}$, Thomas Bengtsson ${ }^{\mathrm{a}}$, Eric Derom ${ }^{\mathrm{b}}$, Romain Pauwels ${ }^{\mathrm{b}}$ \\ a Astra Zeneca $R \& D, S-22187$ Lund, Sweden \\ ${ }^{\mathrm{b}}$ Department of Respiratory Diseases, University Hospital Ghent, De Pintelaan 185, B-9000 Ghent, Belgium
}

Received 11 August 1999; accepted 5 October 1999

\begin{abstract}
In vitro analysis of inhaled formulations measures, among other parameters, the variability in delivered dose, while a corresponding in vivo analysis also includes the variability caused by patient performance and distribution of drug between the oropharynx and the lungs. In vitro, the dose variability is higher for Turbuhaler ${ }^{\circledR}$ than for the corresponding pMDI, whereas in vivo, the converse is true: the variability in lung deposition is significantly higher, both between and within subjects, for pMDI than for Turbuhaler. The observation can be due to several factors such as the non-continuous working principle of inhalation via pMDI as opposed to the continuous working principle of inhalation via Turbuhaler. (C) 2000 Elsevier Science B.V. All rights reserved.
\end{abstract}

Keywords: Inhalers; Variability; Lung deposition; Patients

\section{Introduction}

The amount of drug reaching the effector site determines the elicited effect (Borgström et al., 1996; Pauwels et al., 1997). Thus, for inhaled drugs, it is of interest to evaluate the amount of drug reaching the lungs. Not only the absolute value of, but also the variability in, lung deposition is of interest, as a large variability in lung deposition could be reflected in the exerted effects. The overall delivery of inhaled drugs to the effector site can be described by a number of steps:

\footnotetext{
* Corresponding author.
}

dose metering, aerosol generation, dose delivery (from inhaler to patient), partition of delivered dose between oropharynx and lungs, and, finally, regional deposition of drug reaching the lung. Variability in the different steps will add up to an overall variability in the amount of drug reaching the lungs. The variability in lung deposition thus depends on a large number of in vitro and in vivo factors, e.g. device performance, handling of the device, co-ordination of actuation and inhalation, inhalation flow, patient anatomy etc. In vitro analysis is done to ascertain a good quality of the manufactured product and the analyses are done under strictly standardised conditions. The abso- 
lute amount of drug leaving the inhaler and its variability are typical in vitro parameters. The in vivo analysis includes lung deposition and its variability, but also the clinical outcome of the dose reaching the lungs. The measured in vitro variability thus is only a small portion of the overall in vivo variability observed in the clinical situation.

Lung deposition of inhaled drug can be measured with different methods, gamma-scintigraphy and pharmacokinetic methods being the most widely used (Borgström and Nilsson, 1990; Newman et al., 1995; Pauwels et al., 1997). In the present study the pharmacokinetic charcoal-block method was used. The method takes advantage of the fact that if the uptake of the oral and gastrointestinal portion of an inhaled drug is blocked by activated charcoal, then the amount of active drug reaching the systemic circulation equals the amount of active drug absorbed over the lung membrane. This amount equals the lung deposition. Pharmacokinetic methods are based on measurements of plasma and urine concentrations and thus the variability in the bioanalytical method adds to the overall variability. This methodological variability is probably of minor importance compared with variability in the other factors discussed above. A previous study investigated the variability in lung deposition in healthy volunteers (Beckman et al., 1996). From that study it was concluded that both variability between subjects (intervariability) and variability within subjects (intravariability) in lung deposition were lower for the dry powder inhaler, DPI, Turbuhaler than for a pressurised metered dose inhaler, pMDI.

The aim of the present investigation was to determine the intra-, and intervariability in lung deposition in asthmatic patients when they inhaled terbutaline sulphate (Bricanyl ${ }^{\circledR}$ ) via two different kinds of inhalers, a pMDI and a DPI, Turbuhaler. The results from the previous study in healthy volunteers are given for reference. The present investigation was part of a more extensive study. The main aim of this study was to investigate if differences in the amount of terbutaline sulphate reaching the lungs were reflected in a difference in protective effect against a methacholine or a histamine provocation.

\section{Material and methods}

Thirteen asthmatic patients (nine men) were included in the study. Their mean age was 28 years (range 19-48) and their mean $\mathrm{FEV}_{1}$ was 3.4 L (2.6-4.6), corresponding to $85 \%$ of predicted, at inclusion. Patients were asked to inhale terbutaline sulphate $\left(\right.$ Bricanyl $\left.^{\circledR}\right)$ from a pMDI or Turbuhaler at nominal doses of 0.25 or $0.5 \mathrm{mg}$ and its protective effect against methacholine or histamine provocation was tested. The study was double-blind, crossover and randomised with respect to the provocative agent. The amount of drug reaching the lungs, lung deposition, was calculated at each inhalation, with the aid of a pharmacokinetic method (Borgström and Nilsson, 1990). The $0.5 \mathrm{mg}$ dose was given as two doses of $0.25 \mathrm{mg}$. Thus, the measured lung deposition values, in percentage of dose, for the $0.5 \mathrm{mg}$ doses is the mean of two $0.25 \mathrm{mg}$ doses. For each patient eight determinations of lung deposition were generated, making it possible to calculate the intra-, as well as the intervariability in lung deposition of the inhaled drug. The results from the lung deposition versus effect part of the study have been reported elsewhere (Derom et al., 1999). Lung deposition was compared between devices using a multiplicative ANOVA model with factors patient, visit, device, dose and interaction device by dose. A multiplicative model means that data were analysed on a logarithmic scale. The intraand intervariability in lung deposition with each device was assessed using a linear mixed effects model (LINMEM) accounting for device and visit as fixed factors. Differences in the doses within the same device for asthmatic patients were assumed to be part of the variability. The estimated variances on the logarithmic scale were expressed as coefficients of variation $(\mathrm{CV})$ on the linear scale.

\section{Results}

The absolute amount of drug reaching the lungs differed significantly between the two inhalers (Table 1). The value given for the ratio of Turbuhaler/pMDI is the ratio between the mean 
Table 1

Lung deposition (percentage of nominal metered dose) after inhalation of terbutaline sulphate via pMDI or Turbuhaler in the asthmatic patients group

\begin{tabular}{lccc}
\hline Treatment & Lung deposition (\%) & $95 \%$ conf. lim. (\%) & Range (\%) \\
\hline Turbuhaler 0.25 mg & 20.8 & $16.4-26.6$ & $9.7-54.1$ \\
Turbuhaler 0.5 mg & 16.9 & $13.2-21.7$ & $7.3-50.7$ \\
pMDI 0.25 mg & 4.8 & $3.8-6.1$ & $0.2-15.9$ \\
pMDI 0.5 mg & 7.4 & $5.8-9.5$ & $1.1-53.7$ \\
Turbuhaler/pMDI ratio & 3.14 & $2.46-4.01$ & \\
\hline
\end{tabular}

deposition for Turbuhaler and pMDI, respectively.

The absolute values for Turbuhaler were about three times higher than the pMDI values. This is at accord with a previous study where similar values were observed (Borgström et al., 1996).

The values obtained for inter- and intravariability are given in Table 2.

\section{Discussion}

The intra- and interindividual variability in lung deposition for pMDI and Turbuhaler was previously assessed in 12 healthy subjects in an open, crossover and randomised study (Beckman et al., 1996). A re-calculation of the variability from that study with the LINMEM technique, giving more consistent estimates of the influence of the intravariability on the intervariability, are given together with the results from the present study in Table 2. In the previous study the Turbuhaler and pMDI inhalers were also analysed in vitro. Intra-device variability expressed as $\mathrm{CV}$, was $6.4 \%$ for pMDI and $18.2 \%$ for Turbuhaler, a significant $(P<0.001)$ difference. Also the interdevice variability was significantly higher for Turbuhaler than for pMDI; the ratio of CVs was 2.0 $(P=0.023)$.

It should be observed that in vitro analyses are not primarily designed to predict the in vivo behaviour of a tested formulation. The in vitro analyses are designed to judge if an individual batch of the pharmaceutical formulation complies with the approved specifications. The in vitro analyses can be regarded as a final evaluation of all the steps in the manufacturing process. Thus, one should not expect the in vitro behaviour of an inhaled formulation to be a predictor of its in vivo behaviour unless this has been shown to be the case for a specific set of in vitro/in vivo parameters. This is the case for the relation between fine particle dose (FPD) and lung deposition. It has been shown that FPD, measured with an in vivo like inlet on the impactor, can be used to predict the lung deposition for different DPIs and pMDIs (Olsson et al., 1996).

In the present study, the intravariability in lung deposition in patients was similar for the two devices, while for the healthy subjects it was higher for the pMDI. Taken together this confirms that factors other than the generation of the inhaled aerosol are of importance for the overall variability seen in lung deposition. It also suggests that day to day variability, if anything, is lower for the DPI than for the corresponding pMDI. The resulting lung dose is probably only to a small degree dependent on the variability in the properties of the generated aerosol cloud, and is

Table 2

Inter- and intravariability in lung deposition of terbutaline inhaled via pMDI or Turbuhaler ${ }^{\mathrm{a}}$

\begin{tabular}{lccllc}
\hline Device & \multicolumn{2}{l}{ Patients } & & & \multicolumn{2}{l}{ Healthy volunteers } \\
\cline { 2 - 3 } \cline { 5 - 5 } \cline { 5 - 5 } & Inter & Intra & & Inter & Intra \\
\hline Turbuhaler & 8.17 & 39.3 & & 18.5 & 47.1 \\
PMDI & 61.2 & 39.1 & & 46.7 & 73.0 \\
\hline
\end{tabular}

\footnotetext{
${ }^{a}$ Values are expressed as coefficients of variation (\%). Values for healthy volunteers are given for comparison and are re-calculated from reference (Beckman et al., 1996)
} 
more dependent on the interaction between the aerosol cloud and the oral cavity of the inhaling subject.

The observed difference in intervariability, between pMDI and Turbuhaler, seen both in patients and healthy volunteers, indicates that the performance of Turbuhaler is more subject independent than the corresponding pMDI. In the case of a patient, the lung deposition is more predictable when prescribing Turbuhaler than when prescribing the corresponding pMDI. The observed difference in variability in lung deposition between the DPI Turbuhaler and the pMDI probably represents a class difference between breath actuated DPIs and pMDIs. This is because the generation of aerosol and inhalation is a continuous process with breath actuated DPIs in contrast to pMDIs where aerosol generation and inhalation of the generated aerosol are two distinct processes that need to be co-ordinated by the inhaling subject. This difference can explain the results obtained.

Collectively, we have shown that in an asthmatic patient population the inhalation of terbutaline sulphate from Turbuhaler gives a more reproducible dose to the effector organ, the lungs, than inhalation of an equivalent dose from the corresponding pMDI.

\section{References}

Borgström, L., Derom, E., Ståhl, E., Wåhlin-Boll, E., Pauwels, R., 1996. The inhalation device influences lung deposition and bronchodilating effect of terbutaline. Am. J. Respir. Crit. Care Med. 153, 1636-1640.

Pauwels, R., Newman, S., Borgström, L., 1997. Airway deposition and airway effects of antiasthma drugs delivered from metered-dose inhalers. Eur. Resp. J. 10, 2127-2138.

Borgström, L., Nilsson, M., 1990. A method for determination of the absolute pulmonary bioavailability of inhaled drugs: terbutaline. Pharm. Res. 7, 10681070 .

Newman, S.P., Steed, K.P., Hooper, G., Brickwell, J., 1995. Scintigraphic assessment of the oropharyngeal and nasal depositions of fusafungine from a pressurized inhaler and from a novel pump spray device. J. Pharm. Pharmacol. 47, 818-821.

Beckman, O., Bondesson, E., Asking, L., Källén, A., Borgström, L., 1996. Intra- and interindividual variations in pulmonary deposition via Turbuhaler and a pMDI. J. Aer. Med. 9, 449.

Derom, E., van Schoor, J., Borgström, L., Pauwels, R., 1999. Lung deposition and clinical effect of terbutaline delivered from pMDI and Turbuhaler on airway responsiveness in patients with asthma. Am. J. Respir. Crit. Care Med. 159, A859.

Olsson, B., Borgström, L., Asking, L., Bondesson, E., 1996. Effect of inlet throat on the correlation between measured fine particle dose and lung deposition. In: Dalby, R., Byron, P., Farr, S. (Eds.), Respiratory Drug Delivery V. Interpharm Press, Buffalo Grove, pp. 273281 . 